\title{
Trajectoires socioéconomiques de campus
}

\section{numériques}

Socioeconomic paths of virtual campuses

\section{Patrice Grevet}

\section{(2) OpenEdition}

\section{Journals}

Édition électronique

URL : http://journals.openedition.org/edc/568

DOI : $10.4000 /$ edc.568

ISSN : 2101-0366

Éditeur

Université Lille-3

\section{Édition imprimée}

Date de publication : 1 décembre 2007

Pagination : 73-89

ISBN : 978-2-9514961-8-7

ISSN : $1270-6841$

\section{Référence électronique}

Patrice Grevet, «Trajectoires socioéconomiques de campus numériques », Études de communication

[En ligne], Numéro spécial | 2007, mis en ligne le 01 octobre 2009, consulté le 25 avril 2019. URL

http://journals.openedition.org/edc/568; DOI : 10.4000/edc.568

Ce document a été généré automatiquement le 25 avril 2019.

(c) Tous droits réservés 


\section{Trajectoires socioéconomiques de campus numériques}

Socioeconomic paths of virtual campuses

Patrice Grevet

1 L'ERTe $\mathrm{CN}^{1}$ a étudié divers cas de numérisation de l'enseignement supérieur avec une grille commune afin d'en tirer des lectures transversales. Le présent article relève de celles-ci.

2 Pour la France à laquelle nous nous limitons, les monographies ont porté sur des réalisations qui participèrent aux appels ministériels à "Campus Numériques Français » 2000 à 2002 (appels notés ensuite $\mathrm{CNF})^{2}$. Ces réalisations comportaient a priori un double intérêt. Dans une partie large des disciplines de l'enseignement supérieur, elles représentaient les plus consistantes des introductions du numérique alors engagées. Par ailleurs, les appels à CNF avaient affiché des principes socioéconomiques clairs sur les réalisations qu'ils cherchaient à obtenir: des dispositifs de formation à distance comportant des ressources et un tutorat utilisant le numérique, dispositifs financés principalement par le marché après les impulsions publiques de départ et mis en œuvre par des consortiums d'établissements pour assurer un seuil critique de moyens. La netteté de ces principes fournissait des repères pour l'analyse, par comparaison, du devenir des campus étudiés.

\section{Des monographies et une question transversale}

3 La grille commune préalable aux études monographiques était organisée autour d'une question transversale: «les trajectoires des campus numériques que nous étudions débouchent-elles ou ont-elles en perspective un débouché sur des modèles économiques et des formes organisationnelles tenables dans la durée ?... [Et cela qu'il y ait inscription] dans la reproduction de l'existant ou dans sa modification plus ou moins sensible ». 
4 A posteriori, l'intérêt des cas étudiés dans cette optique a été confirmé. Ces cas illustrent la diversité des «portes» par lesquelles le numérique entre. Dans Forse (sciences de l'éducation) et CampusCultura (conception et management de projets culturels) ${ }^{3}$, le numérique est introduit comme outil de dispositifs de formation à distance; il est utilisé dans le tutorat et pour des ressources pédagogiques relevant essentiellement au début de la «simple mise en accès " (commodités de diffusion des contenus mis dans des formats analogues au monde de l'imprimé et maniables facilement par les personnels universitaires et par les étudiants). Dans le cas de Canège (économie et gestion), il s'agit de dispositifs de formation à distance avec cette fois, dès les débuts, des objectifs de développement systématique de ressources multimédias ${ }^{4}$. L’Umvf (Université médicale virtuelle francophone $)^{5}$ se constitue en éditeur hors marché de ressources numérisées autonomes, et non en organisateur de dispositifs de formation. En mathématiques, physique, chimie, biologie, l'Uel (Université en Ligne) et C@mpuSciences ${ }^{6}$ essayent de coupler une offre de ressources multimédias autonomes et des dispositifs de formation à distance.

5 Un second élément de confirmation a posteriori de l'intérêt des réalisations étudiées tient à la longueur de leurs trajectoires avec un engagement avant les appels à $\mathrm{CNF}$, une vie après ceux-ci, une rencontre de politiques ministérielles différentes, etc.

\section{La contribution à une interprétation d'ensemble de la numérisation du supérieur}

6 En s'appuyant sur la diversité et la durée de ces trajectoires, le présent article propose une réponse à la question centrale posée au début du travail de l'ERTe $\mathrm{CN}$. Cette réponse participe à une interprétation d'ensemble de la numérisation de l'enseignement supérieur schématisable ainsi ${ }^{7}$ :

- De l'enseignement supérieur, avant que la numérisation ne l'affecte dans ses relations avec le monde extérieur et en interne (situation encore très largement présente), nous disons qu'il relève des activités servicielles, activités dans lesquelles des relations entre des personnes, en l'occurrence les enseignants et les étudiants, occupent une place centrale. Cette caractéristique vaut même lorsque les relations fonctionnent de façon unilatérale ou lorsque des personnes ne sont pas connues individuellement;

- Dans le cours actuel de la numérisation de la société, des tendances néo-servicielles commencent à émerger au sein de l'enseignement supérieur. Elles concernent des conditions de la qualité non nécessairement assurées dans les faits. Elles se caractérisent par le rôle renforcé, dans la qualité de l'enseignement supérieur, des interactions vivantes d'enseignants autonomes très qualifiés et des étudiants, par des ressources pédagogiques numérisées muables ${ }^{8}$ intervenant en complémentarité et non en substitution des interactions personnelles. La mise en œuvre cohérente de ces conditions de la qualité pour l'ensemble des étudiants rencontre des obstacles majeurs qui marquent les modalités d'émergence des tendances néo-servicielles. La question du dépassement de ces obstacles commence à être posée, etc.

7 Nous ne prétendrons pas que les données rassemblées dans les travaux de l'ERTe CN démontrent la validité de cette interprétation d'ensemble. Par contre, l'exigence de compatibilité avec ces données a contribué à l'interprétation qui vient d'être évoquée. Plus précisément, ces données nous conduisent à formuler la thèse d'une phase initiale de 
numérisation pédagogique engagée il y a une dizaine d'années et non close à ce jour, thèse qui aurait une portée bien au-delà des cas étudiés par l'ERTe CN.

\section{Une phase initiale de numérisation dans les cadres familiers de l'enseignement supérieur}

Dans cette phase initiale, les possibilités nouvelles que les technologies offrent, et les demandes auxquelles elles poussent, donnent lieu à une numérisation qui progresse, de façon encore modeste, en s'inscrivant dans les cadres socioéconomiques familiers de l'enseignement supérieur, même si les intentions de départ des acteurs étaient différentes, d'où l'intérêt d'adopter une optique de trajectoires. Cela vaut dans les exemples des campus étudiés par différence avec les changements importants que les appels ministériels à CNF avaient projetés.

On constate simultanément, dans cette même phase initiale, des contradictions ${ }^{9}$, des indices, ou des amorces, signalant des éventualités de futurs changements plus profonds, faisant ressortir des obstacles à la concrétisation de telles éventualités, suggérant une pluralité de devenirs possibles. Mais, il nous semble remarquable que ces indices ou amorces s'écartent aussi des principes des appels à CNF.

Les familiers socioéconomiques sont évolutifs et diffèrent selon les pays, les filières, les institutions. L'énoncé de l'intégration du numérique dans l'ordinaire jusqu'à présent n'ignore pas ces évolutions et variétés. Il indique simplement que, dans des contextes déterminés d'enseignement supérieur, les introductions du numérique n'ont pas défini des catégories spécifiques de formation se distinguant nettement des autres du point de vue socioéconomique. La thèse ne néglige pas non plus les efforts d'innovation accomplis par les acteurs de la numérisation; elle n'implique pas que les introductions du numérique n'aient pas constitué des évolutions socioéconomiques. Elle signifie que cellesci ne touchent pas aux caractères socioéconomiques essentiels ou qu'elles sont de même nature que celles intervenues en général dans l'enseignement supérieur sous l'effet de facteurs plus larges.

11 En ce qui concerne l'avenir, il est possible que les recherches de solution aux obstacles qui se présentent actuellement dans les développements de la numérisation poussent à des évolutions qualitativement plus amples. Mais nous ferons l'hypothèse que celles-ci seraient largement dépendantes de transformations intervenant pour des raisons générales liées aux tensions d'ensemble qui affectent l'enseignement supérieur.

12 Pour présenter des éléments empiriques à l'appui de cette thèse, précisons en préalable le contenu mis sous l'expression modèles socioéconomiques d'enseignement supérieur.

\section{Sur les modèles socioéconomiques d'enseignement supérieur}

13 Le projet initial de l'ERTe CN s'interrogeait sur «des modèles économiques et des formes organisationnelles». Dans la mise en œuvre du projet, nous avons été confrontés à l'importance des aspects institutionnels, aux liens étroits entre les coûts, l'organisation du travail, les identités des groupes professionnels. Aussi, il a paru intéressant de 
proposer une approche globale en termes de «modèles socioéconomiques » intégrant les formes organisationnelles.

Un modèle socioéconomique désigne ici un assemblage construit en obtenant une cohérence relative tenable dans la durée et fonctionnant à une échelle qui dépasse le «marginal ». Chacun des éléments assemblés affecte l'ensemble et en porte la marque.

\section{Quatre sous-ensembles interagissant dans un modèle socioéconomique}

Le souci de lisibilité dans la présentation des éléments impliqués dans un modèle socioéconomique conduit à les rassembler en quelques groupes pour ensuite subdiviser ces derniers. Cela peut être opéré de diverses façons. Nous utiliserons une présentation en quatre sous-ensembles interagissant :

- Un type et un niveau d'offre et de demande avec un double aspect, d'une part les valeurs d'usage (les produits ou services du point de vue des effets utiles offerts ou demandés), d'autre part les voies politico-administratives ou de marché par lesquelles les offres et demandes passent, les formes d'intervention des acteurs dans ces voies...

- Une structure de l'activité (structure de "production») caractérisée par les buts qui impulsent cette activité, par des relations entre Travail Vivant et Ressources Informationnelles Objectivées (des précisions sont données plus loin sur le couple TV-RIO), une répartition et une organisation du travail, des identités et rapports de groupes socioprofessionnels, des représentations mobilisatrices des acteurs, un niveau et une structure des coûts...

- Une architecture des institutions et de leur management ;

- Un mode de financement avec de multiples combinaisons possibles entre financement public hors marché, financement privé par les apprenants, financement marchand par des tiers payant privés ou publics, par des fonds collectifs. Le mode de financement inclut les circuits de collecte et d'allocation des fonds par et à diverses institutions et catégories d'acteurs. Il exerce une influence essentielle dans la définition des buts des activités et ses contraintes d'équilibre occupent une place tout aussi essentielle dans la régulation des arrangements socioéconomiques.

16 Nota: Dans les rubriques précédentes, à chaque fois que l'article «Un» est utilisé (dans «Un type », "Une structure », "Une architecture », " Un mode »), il peut recouvrir une configuration de « principes » plus ou moins hétérogènes, voire contradictoires.

17 Cette approche s'inscrit en réaction à des conceptions très répandues identifiant l'économie et le marché ${ }^{10}$. Or, les dimensions économiques hors marché, même lorsqu'elles sont fortement mises en cause aujourd'hui, continuent à occuper une place essentielle dans la formation, numérique inclus : cycles du financement public allant des prélèvements obligatoires vers l'affectation administrative des fonds, puis vers des services dont les prix ne couvrent pas l'ensemble des coûts et qui contribuent à renouveler les bases de création des richesses sur lesquelles les prélèvements obligatoires ultérieurs sont effectués, normes collectives dans le domaine de la formation continue, pratiques de mutualisation, etc.

18 Par ailleurs, l'identification de l'économie et du marché met dans l'ombre les institutions, y compris celles du marché. C'est particulièrement gênant pour l'analyse des expériences qui nous intéressent. Celles-ci s'inscrivent dans des cadres d'action collective fortement 
institutionnalisés; leur devenir dépend d'interactions, de réorientations, d'ajustements " en marchant ", entre acteurs et institutions. D'où a contrario, l'approche proposée ici.

Pour chacun des sous-ensembles d'un modèle présenté ci-dessus, nous allons indiquer quelques manifestations de l'inscription du numérique dans le familier et des indices d'éventuels changements futurs plus profonds.

\section{Offre et demande : l'équilibre entre présentiel et distance}

Les appels ministériels à CNF 2000 et 2001 reposaient sur l'hypothèse d'un développement rapide des marchés de la formation supérieure à distance numérisée. Contrairement à ces représentations, les monographies de l'ERTe $\mathrm{CN}$ suggèrent que les limites habituelles du tout à distance dans le contexte français ne sont pas sensiblement modifiées par l'actuelle numérisation.

21 Il existe bien une demande d'enseignement à distance s'appuyant maintenant sur les apports du numérique. Le campus Forse (Deceuninck, 2005) en témoigne pour les sciences de l'éducation avec aujourd'hui plus de 1000 étudiants inscrits dans une licence ${ }^{11}$ et des masters. CampusCultura (Fichez et Benchenna, 2005) en témoigne aussi à une échelle moindre, sous réserve aujourd'hui de ses difficultés institutionnelles ${ }^{12}$. A contrario, Canège en économie et gestion et C@mpuSciences attirent des effectifs étudiants beaucoup plus faibles relativement aux offres qu'ils présentent. Par exemple, Canège affichait en réponse à l'appel ministériel à projets 2001 un objectif de 1820 étudiants pour 2003-2004 ; cette même année, il a recruté environ 200 étudiants et depuis les effectifs se seraient réduits. La comparaison entre Forse et Canège conduit à retrouver pour le numérique les facteurs habituels favorables à l'enseignement supérieur à distance et leurs limites étroites.

Une première catégorie de facteurs a trait au maillage territorial de l'offre de formation présentielle relativement à la localisation des demandeurs potentiels. Les avantages des interactions personnelles en présentiel ou semi-présentiel l'emportent à chaque fois que les contraintes de localisation ne rendent pas difficiles ces modes. En économie et gestion, le maillage serré de l'offre présentielle donne à celle-ci une assez grande proximité des publics potentiels. Il en va différemment en sciences de l'éducation.

23 Une seconde catégorie concerne la disponibilité temporelle pour la formation. Si la distance et le numérique apportent des avantages de souplesse (avec en contrepartie, moins de stimulation continue par les activités présentielles), ils n'effacent pas les éléments majeurs qui limitent la disponibilité temporelle des salariés ou des autres actifs pour la formation, à savoir les conditions de leur rémunération pendant les temps de formation. Les limites de la disponibilité temporelle sont à rapporter aux difficultés que des formations déterminées présentent pour des actifs relativement à leurs acquis de départ. On peut penser que les matières d'une maitrise de sciences de gestion présentent plus de difficultés pour des personnes de professions intermédiaires en entreprise privée que celles d'une licence en sciences de l'éducation pour des personnels du monde enseignant conduits régulièrement par leur activité à apprendre de façon individuelle.

Les expériences étudiées comportent aussi des indices de la possibilité d'évolutions différentes. Nous les rattachons à l'intérêt porté à l'échelle internationale au «blended learning » (apprentissage mixte) mêlant présence et distance, aux tendances à passer de 
«l'unimodal » au «bimodal », à la diffusion de dispositifs partiellement à distance dans les universités ordinaires concurrençant les établissements spécialisés dans celle-ci ${ }^{13}$. Les indices en question viennent d'orientations qui se sont manifestées dans les appels ministériels à CNF et au sein des universités. L'appel à CNF 2000 posait le principe «d'un accès privilégié de tous les étudiants inscrits dans les établissements d'enseignement supérieur membres du consortium [consortium constituant un campus numérique] aux contenus et aux services développés par le consortium ». Ce principe a été repris sous des formes proches par les appels à CNF ultérieurs. Dans CampusCultura, il a donné lieu à une application limitée avec l'accès à ses cours donné aux étudiants inscrits dans une formation similaire en présentiel à l'université de Provence ou à distance au $\mathrm{CNED}^{14}$. Dans Canège, il a rencontré les préoccupations des présidents des universités du consortium qui, appelés à participer au financement de la production des ressources numérisées, souhaitaient investir au bénéfice de l'ensemble des étudiants de leurs établissements; mais les mises en application n'ont guère dépassé le cas d'auteurs de cours numérisés de Canège réutilisant eux-mêmes ces ressources dans d'autres formations, présentielles ou semi-présentielles. La possibilité de développements plus importants renvoie aux questions posées dans le point suivant.

\section{La structure de l'activité : quelles configurations TV- RIO?}

Concernant les structures d'activité dans les expériences des campus numériques, nous nous centrerons sur les rapports sociaux entre Travail Vivant (TV) et Ressources Informationnelles Objectivées (RIO). Avec les expressions TV et RIO, il s'agit de reprendre, dans les conditions actuelles, la vieille question des relations entre le travail vivant et les moyens objectifs de son exercice (dans une terminologie courante, les rapports entre travail et capital), en ne se limitant pas aux éléments matériels, en prenant pleinement en compte les éléments informationnels objectivés. Le couple TV-RIO serait utilisable dans l'analyse d'une très large gamme d'activités, à chaque fois que de l'information objectivée sur un support matériel joue un rôle important dans la mise en œuvre de l'activité. Précisons le contenu pris par ce couple dans un dispositif de formation.

\section{Sur le couple TV-RIO dans un dispositif de formation}

- Le pôle TV correspond au travail effectué par les participants à une activité de formation au cours d'une période déterminée, d'où sa caractéristique de travail « vivant » relativement à la période considérée. Il est le fait des personnels enseignants, de direction, administratifs, techniques, et des apprenants. Du côté des personnels des organismes de formation, il a pour objet la conception et la gestion des dispositifs de formation, la préparation d'interventions, les rapports directs avec les apprenants ou avec d'autres personnes des institutions auxquels les apprenants appartiennent, diverses tâches de back office (gestion des inscriptions et des dossiers administratifs des apprenants, maintenance des serveurs), etc. Il s'exerce en face à face ou à distance par le téléphone, le courrier, les sites électroniques d'échange...

- Le pôle RIO tient aux ressources informationnelles qui sont objectivées sur des supports matériels (papier, supports numériques ou autres). Il s'agit d'informations sur les formations, de dossiers administratifs des apprenants, de livres, de revues, de polycopiés, de 
plates-formes numériques, de ressources pédagogiques numérisées au sein desquelles on distingue les contenus de formation, les logiciels d'apprentissage et d'évaluation des connaissances, les modes d'emploi des dispositifs d'apprentissage, etc.

Les modalités prises par le couple TV-RIO sont analysables aussi dans la production des ressources pédagogiques numérisées. Pour cette production, des ressources informationnelles préexistantes sont mobilisées et plus ou moins transformées par du travail vivant, d'autres peuvent être construites spécifiquement à cette occasion. Le travail vivant est le fait d'enseignants, d'ingénieurs et de techniciens, au sein et en dehors des institutions de formation.

\section{Un point de départ professionnaliste}

Qu'il s'agisse de la production des ressources pédagogiques ou du fonctionnement des dispositifs de formation, la numérisation participe-t-elle à l'émergence de nouvelles configurations TV-RIO ? Pour répondre à cette question dans les cas étudiés, il faut situer le point de départ tenant à la situation générale de l'enseignement supérieur. Ce point de départ se caractérise par une union TV-RIO, union sous le contrôle des enseignants entre leur travail vivant et les ressources informationnelles objectivées ${ }^{15}$. Deux traits participant à un ensemble de caractères que nous qualifions de " professionnalistes $»^{16}$ sont marquants à cet égard :

- Un accès aux ressources informationnelle objectivées (par exemple les livres) libre et gratuit pour les enseignants ou à des coûts qu'ils sont en mesure de couvrir personnellement;

- Une très large autonomie des enseignants dans le processus de travail signifiant, entre autres aspects, qu'ils contrôlent les usages des ressources informationnelles objectivées dans leurs rapports avec les étudiants, qu'ils ont la possibilité de façonner ces ressources. Ils peuvent avoir rédigé le contenu de celles-ci (polycopiés de cours, livres et articles dont ils sont les auteurs) ou utiliser des ressources réalisées par d'autres, mais en se les appropriant pleinement, sachant qu'une pleine appropriation va de pair avec la latitude de transformer et de fixer des modalités d'usage. Cette situation de départ vaut complètement pour les enseignants responsables de cours et, plus ou moins partiellement, pour les autres membres d'une équipe pédagogique dans des cours à effectifs nombreux comportant des travaux dirigés ou pratiques.

\section{Continuité et amorce de transformations}

28 Les campus numériques étudiés font apparaitre une continuité avec ce point de départ. Dans l'exemple de Canège, les responsables du campus se coordonnent formation par formation pour chercher des enseignants qu'ils estiment compétents et qui acceptent la tâche de rédaction des contenus à numériser. Le dossier déposé en réponse à l'appel ministériel 2000 indiquait qu'un Conseil scientifique dont la composition nominative était précisée validerait le contenu scientifique des cours devant être numérisés. Cette disposition n’a jamais été mise en œuvre. Il n'y a jamais eu non plus de validation de ce type dans les autres campus.

29 Dans Canège, les enseignants-auteurs sont aussi tuteurs. Lorsqu'une ressource est réalisée, les auteurs s'engagent à être tuteurs au minimum lors des deux premières années de mise à disposition de la ressource. D'après les observations effectuées en maijuin 2005, les tuteurs demeurent dans la très grande majorité des cas des auteurs. Ce 
degré élevé de coïncidence entre tuteurs et auteurs est influencé par le nombre limité des étudiants de Canège. Mais, il est remarquable que Canège ait toujours affirmé une règle générale d'autonomie des tuteurs, qu'ils soient auteurs ou non. Chacun d'entre eux est le responsable du module pour les étudiants vis-à-vis desquels il intervient ; il est aussi celui qui note. Lorsque le terme "tuteur» est employé, il désigne toujours un enseignant exerçant une fonction tutorale et non pas une catégorie distincte des enseignants habituels des universités. L'orientation de Canège est celle de tuteurs s'appropriant les ressources numérisées lorsqu'ils n'en sont pas les auteurs, en usant avec liberté, y ajoutant les éléments qu'ils souhaitent.

Dans d'autres campus, nous n'avons pas une affirmation aussi tranchée de la responsabilité et de l'autonomie des tuteurs qu'ils soient auteurs ou non. Mais, en termes de division du travail, pour des cours à effectifs nombreux, par exemple la licence de Forse, il ne semble pas qu'on aille au-delà de ce qu'on avait connu depuis les années 1960, ou connaissait encore, dans les cours présentiels à très gros effectifs de différentes disciplines, avec des chargés de TD ou TP distincts de l'enseignant responsable du cours. A cet égard, nous ne voyons pas de traces notables d'industrialisation dans les campus numériques étudiés.

Elisabeth Fichez, dans une lecture transversale des monographies de l'ERTe CN, note une large continuité avec les démarches professionnelles habituelles: faiblesse des contraintes liées aux normes et standards dans la production des ressources, styles d'auteur hétérogènes dans les scénarios pédagogiques et les consignes d'usage des ressources (Fichez, 2006a et b). Ajoutons qu'il pourrait y avoir un plus grand degré de standardisation des mises en forme ou des types de documents pédagogiques (texte intégral de cours, glossaire, présentation vidéo, liens hypertextes, etc.) sans que cela touche à l'essentiel dans l'union TV-RIO.

Quant à la rédaction complète des cours et à leur diffusion aux étudiants en principe avant le début d'un module de formation, elles représentent bien des modifications des pratiques professionnelles dominantes: modification de la charge de travail, accroissement des exigences de mise au point induites par la visibilité des cours aux yeux des étudiants, des collègues, ou d'autres personnes, etc. Mais reste à apprécier la portée socioéconomique de ces modifications.

Pour ce faire, rappelons l'antériorité de polycopiés complets de cours engageant la responsabilité des enseignants dans des formations habituelles. Par exemple, à Paris, depuis les années 1950, s'était établie une norme dominante de polycopiés payants à la Faculté de droit et sciences économiques, à l'IEP (Institut d'Études Politiques); à un moindre degré, c'était aussi le cas dans d'autres disciplines de sciences sociales et humaines. Ces polycopiés étaient produits par des associations étudiantes ou par des entreprises privées à partir de la sténographie des cours prononcés, et après correction par les professeurs responsables. La généralisation des polycopiés complets et leur gratuité constituèrent une revendication étudiante en mai-juin 1968. La situation a plutôt évolué vers des cours à effectifs moins grands avec des pratiques très partielles de polycopiés fournis gratuitement par les services universitaires.

Aujourd'hui, la réalisation et la diffusion numériques des cours et de matériaux associés, avant les interactions avec les étudiants d'une promotion donnée, peuvent correspondre à des modifications importantes de la pédagogie, des formes de travail des enseignants et des étudiants. Mais cela n'implique pas en soi la disparition des deux traits professionnalistes de départ (l'union TV-RIO) indiqués ci-dessus. Nous en avons un 
exemple à la Faculté de médecine de Grenoble qui, depuis la rentrée 2006, diffuse aux étudiants de première année les cours sur DVD et organise la formulation en ligne des questions sur les cours avant des séances d'enseignement explicatif ${ }^{17}$.

E. Fichez (2006a), à la suite de J. Deceuninck (2005, p. 25), s'interroge sur l'amorce de rupture que pourrait représenter la rémunération d'enseignants en tant qu'auteurs dans des campus numériques. Il nous semble qu'il s'agit d'un prolongement de schémas de rémunérations complémentaires habituels dans les rapports avec les éditeurs ou divers organismes privés. Certes, la rémunération complémentaire, en tant qu'auteur, a la particularité ici de pouvoir être interne à l'institution dans laquelle des enseignants sont en poste (cas de Canège sous des formes qui ont été négociées pour être acceptables par les agents comptables, sans que leur solidité juridique soit très assurée) ${ }^{18}$, alors que dans le cas de Forse, la rémunération est versée par le $\mathrm{CNED}^{19}$. Mais on est clairement dans le compromis avec des enseignants très attachés au droit d'auteur dans ses formes classiques et entendant le négocier contre une rémunération spécifique.

Les amorces de changements importants nous semblent plutôt se situer du côté de l'intégration dans "le travail normal de l'enseignant-chercheur ${ }^{20}$ de pair avec l'avancée de normes du libre. Des enjeux importants se nouent là concernant la charge de travail des enseignants, la définition globale de leurs services, les coûts temporels de la réutilisationtransformation des ressources numériques par les enseignants, les collaborations avec d'autres catégories professionnelles, etc. Ces enjeux nous semblent donc se situer dans des cadres professionnalistes plus ou moins transformés ${ }^{21}$.

\section{Architecture des institutions et management}

Sur ce point, nous serons très bref en renvoyant aux articles d'Elisabeth Fichez déjà cités. Nous partageons sa lecture transversale : forte continuité dans le management relevée en notant que "le pilotage des projets de campus n'a pas donné lieu à l'apparition d'une 'couche' managériale autonome d'acteurs spécifiquement recrutés pour la circonstance» (Fichez, 2006a), mise en cause de l'originalité institutionnelle voulue par les appels à CNF avec des tendances à la décomposition des consortiums de dispositifs de formation (ibid. et Fichez, 2006b). Quant à la question des amorces de changements durables affectant les institutions et le management, nous la reprendrons en conclusion.

\section{Des modes de financement habituels}

La tendance à l'intégration dans le familier est aussi frappante à propos du financement. Dans les expériences de certains campus numériques, des forces poussent à accroître les droits d'inscription demandés aux apprenants ou à des tiers payants; les appels ministériels à CNF escomptaient ainsi à terme un financement tiré principalement d'une forte croissance des marchés de la formation à distance. Mais, dans les faits, cela n'a pas été différent des pressions à l'augmentation des droits qui s'exercent de façon générale de l'intérieur des universités face aux limites des financements publics et aux opportunités que les marchés présentent, pressions contenues sous l'influence des organisations étudiantes et du contexte politique national jusqu'à présent et peut-être aussi à l'avenir ${ }^{22}$. Le financement des dispositifs de formation étudiés par l'ERTe CN s'est inscrit dans la distinction habituelle entre formation initiale à financement essentiellement public et 
formation continue payante faisant largement intervenir des tiers financeurs. En formation initiale, l'idée présente au début des années 2000 que les avantages du numérique et d'Internet s'imposeraient aux yeux des étudiants, qu'ils feraient accepter des tarifs plus élevés ${ }^{23}$ que dans les formations à distance papier classiques, a été démentie. En formation continue, les tarifs pratiqués sont du même ordre que les prix habituels de marché.

Quant aux investissements en ressources multimédias coûteuses (Université en ligne dont les ressources sont utilisées par C@mpusciences, UMVF, Canège), ils ont été couverts par la conjonction de financements publics directs, de dépenses importantes de temps d'enseignants ou d'autres catégories pionniers, dépenses non comptabilisées monétairement, mais adossées sur le financement public statutaire des postes des personnels impliqués. Dans le cas de Canège, il s'y est ajouté un important apport du CNED, organisme public se plaçant en la matière dans une perspective de retour sur investissement qui n'a d'ailleurs pas eu lieu.

\section{Conclusion : tensions gestionnaires et sous- financement}

41 En conclusion, nous évoquerons les indices d'éventuels futurs changements importants qui seraient décelables dans l'expérience des campus numériques sous l'angle des institutions, du management, du financement. Il nous semble que ces indices s'inscrivent pleinement dans des problèmes généraux rencontrés aujourd'hui par l'enseignement supérieur en France et donc que les suites qui y seraient données se feraient sous dépendance de changements dépassant de loin les aspects liés directement au numérique.

La numérisation de l'enseignement constitue un facteur d'accroissement, parmi bien d'autres, de la complexité des organisations du supérieur. Elle appelle un développement de coordinations souples et très évolutives entre activités et catégories professionnelles. Des études de cas réalisées par l'ERTe CN montrent des difficultés tenant à des insuffisances des institutions et du management universitaires en matière de logistique, d'inscription réactive des étudiants potentiels en formation à distance et/ou continue, de gestion cohérente de tous les aspects d'un projet innovant. Elles montrent les inconvénients de solutions qui ont été recherchées dans l'externalisation de certaines fonctions auprès du CNED, y compris la gestion de flux financiers stratégiques de pair avec la non stabilisation sur postes universitaires de dispositifs qui réussissent pourtant à attirer de nombreux étudiants. Ce type de problèmes participe de difficultés d'ensemble face au développement des fonctions gestionnaires dans les établissements d'enseignement supérieur.

Simultanément, l'enseignement supérieur français est largement sous-financé. La prise en charge des coûts des interactions personnelles, du développement et de la réutilisation-transformation des ressources pédagogiques dans des processus de formation numérisés se heurte à ces difficultés d'ensemble.

L'hypothèse que nous confronterons à de nouvelles études de cas est que les combinaisons entre traits professionnalistes et tendances à la montée du managérialisme ${ }^{24}$ peuvent être très variables. Un facteur essentiel dans les évolutions qui interviendront à cet égard tiendrait aux solutions qui seront en même temps recherchées face aux tensions sur le financement de l'enseignement supérieur. Il faut en effet souligner que la 
montée du managérialisme a été associée à l'échelle internationale, pendant les deux dernières décennies, au développement de la concurrence entre institutions du supérieur, notamment pour les ressources financières.

\section{BIBLIOGRAPHIE}

Casanova, J.-C., (2006), « Une nouvelle chance pour les universités », Le Monde, 22 décembre 2006.

Combès, Y. et Mœglin, P., (2005), C@mpuSciences d'un modèle industriel à l'autre, accessible aux adresses indiquées à ERTe CN 2005.

Deceuninck, J., (2005), Campus Forse, Formation et Ressources en Sciences de l'éducation, accessible aux adresses indiquées à ERTe CN 2005.

ERTe CN, (2005), Résultats 2005 de l'ERTe (Équipe de Recherche Technologique éducation) « Modèles économiques et enjeux organisationnels des campus numériques ", accessible à www.ifresi.univlille1.fr/SITE/2_Recherche/22_Programmes/ERTe/ERTe.htm ou à partir de la page d'accueil www.mshparisnord.org/.

Fichez, é. et Benchenna, A., (2005), Le cas CampusCultura, accessible aux adresses indiquées à ERTe CN 2005.

Fichez, é., (2006a), « Campus numériques : des ambitions à l'épreuve des terrains », Distances et Savoirs, volume $4, \mathrm{n}^{\circ} 3 / 2006$.

Fichez, é., (2006b), « Campus numériques français : pertinence des notions de réussite ou d'échec », Études de communication, numéro spécial 2007.

Grevet, P., (2005a), Socio-économie de l'information et des services interpersonnels. Une approche de l'enseignement supérieur utilisant le numérique. Note de recherche, Ifrési, Contrat de Plan Cnrs Région Nord/Pas-de-Calais, http://www.univ-lille1.fr/clerse/site_clerse/pages/accueil/fiches/ Grevet.htm.

Grevet, P., (2005b), L'expérience socio-économique de Canège, accessible aux adresses indiquées à ERTe CN 2005.

Grevet, P., (2006a), « Une contradiction structurante dans la numérisation de l'enseignement supérieur », Distances et Savoirs, volume 4, nº 3/2006.

Grevet, P., (2006b), « Le numérique dans l'enseignement supérieur : des tendances néoservicielles ", Éducation permanente, n 169, 2006-4.

Horn, F. et Lamarche, T., (2005), L'Université Médicale Virtuelle Francophone (UMVF). Accessible aux adresses indiquées à ERTe CN 2005.

Perraton, H., (2006), « Éducation supérieure virtuelle : l'expérience du Common-wealth », Éducation permanente, $\mathrm{n}^{\circ} 169,2006-4$. 


\section{NOTES}

1. Équipe de Recherche Technologique éducation «Modèles économiques et enjeux organisationnels des campus numériques » (sur ses résultats, voir ERTe CN 2005).

2. Pour des précisions sur la représentativité des cas étudiés par l'ERTe CN, $c f$. Grevet, 2006a.

3. Pour Forse, voir Deceuninck, 2005, et pour CampusCultura, Fichez et Benchenna, 2005.

4. Cf. Grevet, $2005 \mathrm{~b}$.

5. Cf. Horn et Lamarche, 2005.

6. Cf. Combès et Mœglin, 2005.

7. Pour une présentation plus détaillée de cette interprétation, voir Grevet, 2006b.

8. Nous proposons les termes muable et muabilité à propos de ces ressources pour souligner leur potentiel d'ouverture à des modifications permanentes liées à l'usage en rupture avec les caractéristiques des produits industriels pour lesquels la part de la reproduction reste importante. En exploitant des potentialités du numérique, on passe de la reproductibilité coûteuse à la muabilité coûteuse.

9. Dans Grevet, 2006a, nous avons souligné une contradiction structurante marquant la numérisation de l'enseignement supérieur en France depuis une dizaine d'années : tendance à la polarisation sur l'offre de ressources pédagogiques numérisées, et usages étroitement limités de ces ressources dans les dispositifs institutionnalisés de formation.

10. De telles connotations se trouvent dans des usages fréquents de l'expression «trouver son modèle économique " pour une activité sur Internet, avec une assimilation à l'idée de "business model » traduisible par « modèle d'affaires (sous-entendu commerciales) » ou de "modèle d'entreprise (sous-entendu, là aussi, commerciale)».

11. La licence est l'équivalent d'une formation présentielle en un an comportant des prérequis de type Bac +2 .

12. Après le retrait de l'université d'Avignon et celui du CNED, le devenir de CampusCultura repose depuis la rentrée 2005-2006 sur la seule université de Provence.

13. Concernant la formation mixte dans les pays du Commonwealth, voir Perraton, 2005.

14. Cf. Fichez et Benchenna, 2005, pp. 21-22.

15. Cette union se définit par opposition à une séparation-réunion sous une autorité extérieure dont la forme extrême serait la suivante : séparation entre un formateur et les conditions objectives de son activité déterminées entièrement en dehors de lui, puis réunion dans le processus de travail par une autorité prescrivant notamment au formateur les ressources pédagogiques à accompagner et les modalités de l'accompagnement.

16. Pour des développements sur le sens que nous donnons à ce terme, voir Grevet, 2005a, pp. 21-22 et 26-27.

17. Cf. www-sante.ujf-grenoble.fr/SANTE/medatice/.

18. Cf. Grevet, 2005b, pp. 49-51.

19. Cf. Deceuninck, 2005, p. 25.

20. Cf. Fichez, 2006a reprenant une formule mise en avant dans l'UMVF (voir Horn et Lamarche, 2005).

21. Pour des précisions à ce propos, $c f$. Grevet, 2006a et 2006b, pp. 161-166.

22. Cf. par exemple Le Monde des 19 et 25 juillet 2006 et, en ce qui concerne la possibilité que ces pressions restent contenues à l'avenir, Casanova, 2006.

23. Avec en outre les frais personnels en équipements informatiques, connexion Internet et impression papier.

24. Le managérialisme évoqué concerne l'organisation et l'exercice des pouvoirs dans la vie des institutions de l'enseignement supérieur. Il s'affirme lorsque, dans les choix stratégiques 
d'organisation des formations et dans la gestion courante, une place dominante est prise par des managers dont les carrières se séparent nettement de celles des enseignants-chercheurs. Il interagit avec un système de contraintes et incitations extérieures tenant aux rapports avec les pouvoirs publics et les marchés.

\section{RÉSUMÉS}

L'article s'attache aux trajectoires de campus numériques français et aux indications qu'elles fournissent sur les modèles socioéconomiques d'enseignement supérieur. Ces indications participent au développement d'une lecture néo-servicielle de la numérisation de l'enseignement supérieur (évolution des conditions de la qualité avec le rôle renforcé des interactions enseignants-étudiants et la complémentarité entre ces interactions et des ressources numérisées muables, obstacles à la mise en œuvre des conditions actuelles de la qualité...). Elles conduisent à formuler la thèse d'une phase initiale de numérisation pédagogique engagée il y a une dizaine d'années et non close à ce jour. Dans cette phase, la numérisation progresse, de façon encore modeste, en s'inscrivant dans les cadres socioéconomiques familiers de l'enseignement supérieur, avec simultanément des contradictions, des indices, ou des amorces, signalant des éventualités de futurs changements plus profonds. L'article souligne que ces futurs changements dépendent de transformations beaucoup plus générales liées aux tensions d'ensemble qui affectent l'enseignement supérieur, notamment en termes de gestion et de financement face aux conditions actuelles de la qualité.

This article examines the various directions found in French virtual campuses and the potential indicators they reveal with regard to higher education socioeconomic models. The collected data contribute to developing a "neo-servicial » understanding of digitization in higher education (teaching quality being dependent on reinforced live interactions between teachers and students, possibilities offered by digitised «muable» resources being used in conjunction with, and not instead of, interpersonal exchanges, obstacles to implementation of theses conditions of effectiveness of learning, etc.). Within framework, the analysis of campuses leads the author to the thesis that an initial phase of teaching digitization exists. This stage started ten years ago and is still incomplete. In this stage digitization has been slowly increasing within the existing socioeconomic framework of higher education in France and is marked by simultaneous contradictions, and beginnings that can signal potential deeper future changes. The article underlines that these future changes are dependent on much broader modifications, linked to financing and management issues in French higher education, in particular in view of present quality conditions.

\section{INDEX}

Mots-clés : campus numérique, modèle socioéconomique, ressources numériques, enseignement supérieur, management

Keywords : virtual campus, socioeconomic model, digital resources, higher education, management 


\section{AUTEUR}

\section{PATRICE GREVET}

Patrice Grevet est professeur émérite en sciences économiques à l'Université Lille 1. Il est membre du Clerse (Centre lillois d'études et de recherches sociologiques et économiques, Umr Cnrs-Université Lille 1) et de l'Ifrési (Institut fédératif de recherche sur les économies et les sociétés industrielles, Fédération de recherche du Cnrs). Ses travaux de recherche portent sur la socioéconomie de l'enseignement supérieur et de la formation continue. Il développe notamment une problématique néo-servicielle de la numérisation de l'enseignement supérieur. Page personnelle : http://www.univ-lille1.fr/clerse/site_clerse/pages/accueil/fiches/Grevet.htm. Adresse électronique : patrice.grevet@ifresi.univ-lille1.fr. 